\title{
Disentangling neural structures for processing of high- and low-speed visual motion
}

\author{
Jeannette A. M. Lorteije, ${ }^{1,2,3}$ Richard J. A. van Wezel ${ }^{1}$ and Maarten J. van der Smagt ${ }^{2}$ \\ ${ }^{1}$ Functional Neurobiology and \\ 2Experimental Psychology, Helmholtz Institute \& Utrecht University, Heidelberglaan 2, 3584 CS Utrecht, the Netherlands \\ ${ }^{3}$ Department of Neuroscience, Erasmus MC, the Netherlands
}

Keywords: adaptation, human, motion vision, speed tuning, visual evoked potentials

\begin{abstract}
Human psychophysical and electrophysiological evidence suggests at least two separate visual motion pathways, one tuned to a lower and one tuned to a broader and partly overlapping range of higher speeds. It remains unclear whether these two different channels are represented by different cortical areas or by sub-populations within a single area. We recorded evoked potentials at 59 scalp locations to the onset of a slow $\left(3.5^{\circ} / \mathrm{s}\right)$ and fast $\left(32^{\circ} / \mathrm{s}\right)$ moving test pattern, preceded by either a slow or fast adapting pattern that moved in either the same direction or opposite to the test motion. Baseline potentials were recorded for slow and fast moving test patterns after adaptation to a static pattern. Comparison of adapted responses with baseline responses revealed that the N2 peak around $180 \mathrm{~ms}$ after test stimulus onset was modulated by the preceding adaptation. This modulation depended on both direction and speed. Source localization of baseline potentials as well as direction-independent motion adaptation revealed cortical areas activated by fast motion to be more dorsal, medial and posterior compared with neural structures underlying slow motion processing. For both speeds, the direction-dependent component of this adaptation modulation occurred in the same area, located significantly more dorsally compared with neural structures that were adapted in a direction-independent manner. These results demonstrate for the first time the cortical separation of more ventral areas selectively activated by visual motion at low speeds (and not high speeds) and dorsal motion-sensitive cortical areas that are activated by both high and low speeds.
\end{abstract}

\section{Introduction}

Motion processing is vital for most animals to interact with a dynamic environment, whether for detecting moving prey, predators or partners, for crossing streets without being hit by a car, or to intercept a moving object. Even though we as observers might experience low and high speeds as aspects of a continuous spectrum, human psychophysical experiments have given rise to the idea of two separate motionprocessing pathways, one channel that is tuned to low temporal frequencies and high spatial frequencies (low speeds) and one channel that is tuned to a large bandwidth of high temporal frequencies and low spatial frequencies (high speeds) (Kulikowski \& Tolhurst, 1973; Thompson, 1984; Anderson \& Burr, 1985; Smith \& Edgar, 1994; Gegenfurtner \& Hawken, 1996; van de Grind et al., 2001). [However, see van Boxtel et al. (2006), who postulate that a single motion channel may explain the entire range of perceived speeds.]

Several human psychophysical studies used motion adaptation to study characteristics of these two channels. More specifically, these studies focused on the motion aftereffect (MAE) (for a review see Mather et al., 1998), a visual illusion caused by motion adaptation. After prolonged exposure (i.e. at least several seconds) to a pattern moving in a single direction, a stationary pattern may be experienced as moving in the opposite direction to the adapting motion. Interestingly, the speed of the adapting pattern and refresh rate of the test pattern interact. Whereas low speeds are capable of evoking an

Correspondence: Dr Maarten J. van der Smagt, as above.

E-mail: M.J.vanderSmagt@uu.nl

Received 28 June 2007, revised 13 February 2008, accepted 6 March 2008
MAE on static test patterns or patterns at low refresh rates (static MAE), fast motion evokes an MAE on patterns with high refresh rates (like 'snow' on a detuned television; dynamic MAE) (Verstraten et al., 1998, 1999). These results show that the different channels for lowand high-speed motion can be adapted separately. The divergence between both channels does not simply reflect a difference in processing of two extremes of a single continuous speed or temporal frequency range as, after simultaneous adaptation with a transparent fast- and slow-moving pattern, a sharp turnover point in MAE direction is observed at test pattern refresh frequencies of approximately $20 \mathrm{~Hz}$. Furthermore, when the test pattern consists of combined low and high temporal frequencies it is even possible to perceive a transparent MAE (van der Smagt et al., 1999).

Recording of visually evoked potentials (VEPs) with electroencephalography (EEG) has become a valuable tool for studying physiological structures underlying motion processing. Motion onset evokes a typical negative peak with a latency of 150-200 ms, which is often referred to as N2 or N200 (Probst et al., 1993; Kubova et al., 1995; Niedeggen \& Wist, 1998; Hoffmann et al., 1999; Lorteije et al., 2006). Motion adaptation was used to study the processes underlying this N2. Preceding motion onset with an adapting motion resulted in a decrease of the N2 amplitude, which is evidence that this peak mainly reflects motion processing (Bach \& Ullrich, 1994). Part of this reduction was direction dependent, i.e. it occurred for test directions approaching the adapting motion direction but not for test directions opposing the adapting direction (Bach \& Hoffmann, 2000; Hoffmann et al., 2001; Heinrich \& Bach, 2003). Heinrich et al. (2004) combined low and high adaptation speeds with low and high test speeds in the 
opposite or same directions. Adaptation was not only direction dependent but also speed specific. This reduction was in accordance with the speed dependence of the MAE and provided physiological evidence for the existence of two separate speed channels. However, as they used only three electrodes in their recordings, the authors could not perform a source localization to establish whether the two channels involved different anatomical regions.

To investigate whether a two-channel system is divided across anatomical structures, we recorded VEPs from 59 scalp positions for the baseline as well as adapted N2s, at both low and high speeds. Together, these electrodes showed the distribution of the N2 across the scalp, for slow and fast baseline recordings, as well as adapted $\mathrm{N} 2 \mathrm{~s}$ and difference potentials between conditions. The scalp distribution of a VEP is dependent on the location and orientation of the underlying neural structures in the brain. Brain electric source analysis (BESA) modelling can be performed on these distributions to estimate the relative location of the cortical areas responsible for these scalp distributions. A number of other studies have also localized VEP responses to motion, using a variety of methods (e.g. Probst et al., 1993; ffytche et al., 1995, 2000; Buchner et al., 1997; Wang et al., 1999; DelonMartin et al., 2006). Most of these, however, focus on specific areas such as V5 or the primary visual area and/or time of arrival of the motion signal in these areas. None, so far, have focused on motion speed.

As a starting point we first analysed possible adaptation effects on the N2 amplitude both within and across high and low speeds, as Heinrich et al. (2004) had already demonstrated. After (partial) success, we set out to determine the dipole sources of the N2 for both speeds and whether they differed in location. Subsequently, we tried to disentangle which (if any) N2 sub-components after different adaptation regimes were responsible for this location difference.

\section{Materials and methods}

\section{Subjects}

A total of 17 subjects participated in this study (six males and 11 females). One male and one female author participated as subjects but all other 15 subjects were naive as to the purpose of the study. All participants gave their written informed consent and the purpose of the experiment was explained to them after the recordings. Naive subjects were paid expenses. Experiments were in accordance with the declaration of Helsinki (World Medical Association 2000) and the protocol was approved by the ethical committee of the faculty of Social and Behavioural Sciences of Utrecht University.

All subjects had normal or corrected-to-normal visual acuity and all subjects were right-handed except for one female. The average age was 22.2 years (SEM 1.2 years).

\section{Stimuli}

Stimuli were presented using Presentation software (Neurobehavioural Systems, Albany, CA, USA) on a 19 inch LaCie electronblue IV monitor $(1024 \times 768$ pixels $)$ with a refresh rate of $85 \mathrm{~Hz}$ at a distance of $57 \mathrm{~cm}$. The stimuli and their presentation were made to match those used by (Heinrich et al., 2004).

A random pixel array (RPA) with a pixel size of $0.04^{\circ}$ moved within a round aperture of $24^{\circ}$ diameter. The contrast of the RPA was $73 \%$, at which the space-averaged luminance was $18 \mathrm{~cd} / \mathrm{m}^{2}$. In order to reduce optokinetic nystagmus, a relatively large fixation target $\left(3^{\circ}\right.$ in diameter) was centred on the pattern.
The RPA could move in either a leftwards or rightwards direction at $3.5^{\circ} / \mathrm{s}$ (slow) or $32 \%$ s (fast). These speeds matched those used in the experiment of Heinrich et al. (2004) and were chosen because they were within the speed ranges that are processed by mostly slow or by exclusively fast motion-processing channels, respectively (Verstraten et al., 1998, 1999; van der Smagt et al., 1999; van de Grind et al., 2001).

Trials were presented in a cyclic design, starting with an adaptation period of $2200 \mathrm{~ms}$ followed by an interstimulus interval of $500 \mathrm{~ms}$ and finally a test period of $300 \mathrm{~ms}$. During the adaptation period the RPA could move at either low or high speed in a leftwards or rightwards direction, or could remain stationary for the baseline recordings. The duty cycle of the test stimulus (test duration as a percentage of the total trial duration) was $10 \%$ to keep the effects of motion adaptation by the test stimulus to a minimum (Bach \& Ullrich, 1994; Hoffmann et al., 1999).

Trials were presented in single-adaptation-type blocks (200 trials per block, except for one subject who was presented with only 150 trials per block). This 'top-up' adaptation ensured a deep adaptation state throughout each block. To ensure a deep adaptation at the first trials of each block, every block was preceded by $30 \mathrm{~s}$ of non-stop adaptation. Subjects were instructed to take a short break of at least 3 min between blocks to prevent fatigue and transfer of adaptation onto the next block. Blocks were presented in a counterbalanced block design, e.g. slow left adaptation, fast right adaptation, a double baseline block, fast left adaptation and slow right adaptation. The condition that was presented in the first block (which decided the order of the following blocks) was counterbalanced between subjects.

During the interstimulus interval, the RPA remained stationary for $500 \mathrm{~ms}$ and started moving again at the onset of the test period. The test pattern could move either fast or slow and rightwards or leftwards, regardless of the adaptation speed and direction. As responses to leftwards and rightwards motion cannot be distinguished in the motion VEP, trials were not discriminated on test motion direction per se. Instead, trials were grouped according to the congruence of the adaptation and test direction, i.e. test motion in either the same or the opposite direction as the adaptation direction (Table 1).

\section{Recordings}

The recordings were performed in a darkened room. A chin rest was placed in front of the monitor to ensure that subjects remained at a viewing distance of $57 \mathrm{~cm}$ throughout the recordings. The EEG was recorded from $59 \mathrm{Ag} / \mathrm{Cl}$ ring electrodes, which were mounted in an elastic cap (Braincap, Brain Products, Germany). Scalp electrodes were distributed according to a 10/10 system. Additional electrodes included two electrodes above and below the left eye to record the

TABLE 1. Combinations of direction and speed of the adaptation and test motions, resulting in 10 conditions (right column)

\begin{tabular}{lll}
\hline Adaptation speed & Test speed & Test direction* \\
\hline Static (baseline) & Fast & n/a \\
\multirow{2}{*}{ Fast } & Slow & n/a \\
& Fast & Opposite \\
& Fast & Same \\
Slow & Slow & Opposite \\
& Slow & Same \\
& Fast & Opposite \\
& Fast & Same \\
& Slow & Opposite \\
& Slow & Same \\
\hline
\end{tabular}

*Compared with adaptation direction. $\mathrm{n} / \mathrm{a}$, not applicable. 
vertical electro-oculogram, two electrodes on the outer canthi of both eyes for horizontal electro-oculogram and one grounding electrode, located between Fpz and AFz. Resistance between skin and electrodes was kept below $4 \mathrm{k} \Omega$ throughout the experiment. A BrainVision Recorder (Brain Products) was used to sample and digitize the EEG at $1 \mathrm{kHz}$, filter (high-pass cutoff at $0.03 \mathrm{~Hz}$, low-pass cutoff at $400 \mathrm{~Hz}$ and a $50 \mathrm{~Hz}$ notch filter), and to store the data on hard disk for off-line analysis. Electrode $\mathrm{Cz}$ was used as reference during the recordings. Data were re-referenced offline to the average scalp potential for further analysis.

\section{VEP analysis}

A BrainVision Analyser (Brain Products) was used for data analysis. The EEG was segmented into stimulus-locked epochs of $500 \mathrm{~ms}$ (100 ms before to $400 \mathrm{~ms}$ after test stimulus onset). Segments containing blinks or eye movements (criteria were $>100 \mu \mathrm{V}$ or $<-100 \mu \mathrm{V}$ on the electro-oculogram channels), or artefacts (e.g. due to movements of the jaw; criteria were $>120 \mu \mathrm{V}$ or $<-120 \mu \mathrm{V}$ on any EEG channel) within a time window of $100 \mathrm{~ms}$ before to $100 \mathrm{~ms}$ after the test period were excluded from further analysis. For every subject, the average evoked potential per condition was calculated. Finally, average VEPs were filtered (high-pass cutoff $0.05 \mathrm{~Hz}$, low-pass cutoff $20 \mathrm{~Hz}, 12 \mathrm{~dB} / \mathrm{oct}$ ) and baseline corrected (i.e. VEPs from all scalp electrodes were offset corrected so that their average potential from 100 to $0 \mathrm{~ms}$ before stimulus onset was $0 \mu \mathrm{V}$ ).

The EEG potentials evoked by motion are most pronounced at occipital and occipito-parietal electrodes (Probst et al., 1993; Bach \& Ullrich, 1994; Hoffmann et al., 2001; Heinrich et al., 2004; Lorteije et al., 2006). For this reason, eight posterior electrodes were selected for analysis on peak amplitudes (PO7, PO3, POz, PO4, PO8, O2, Oz and $\mathrm{O} 1$; see Fig. 4 for scalp locations of these electrodes). For every subject the N2 peak values and corresponding latencies were established at these electrode locations. As the N2 is often lateralized to the left or right hemisphere across subjects (Andreassi \& Juszczak, 1982), for every subject the two electrodes (or single electrode if slow and fast motion responses were maximal at the same electrode position) with maximal $\mathrm{N} 2$ amplitude for slow and fast motion were selected for further analysis. To assess modulation of this N2, the baseline N2 needed to be strong enough. Therefore, an acceptance criterion was set. Only subjects with both high- and low-speed baseline $\mathrm{N} 2$ amplitudes more negative than $-2 \mu \mathrm{V}$ were included in further analysis. Only one subject was excluded based on this criterion.

\section{Source analysis}

To locate the neuronal sources underlying fast and slow motion processing, BESA (BESA 2.2, Scherg \& Picton, 1991) was performed. The analysis included data from all scalp electrodes (not just the eight posterior electrodes). BESA modelled the location, orientation and strength of equivalent intracranial dipole sources according to the recorded scalp activity. The optimal dipole solution was found by searching for a minimum in the residual variance (RV) function in a $3 \mathrm{~ms}$ window at the fast and slow latencies of the baseline (either for grand average or for individual subjects). To reduce the probability of interacting dipoles (i.e. adjacent dipoles with opposing high-amplitude potential fields), the energy constraint of the BESA model was set to $20 \%$ (with the remaining $80 \%$ for the RV criterion), thus favouring source solutions with relatively low dipole strengths (Berg \& Scherg, 1994). Single dipole pairs were used for source models. The location and orientation of the dipoles were bilaterally symmetrically constrained.

None of the BESA models in this study had an RV above $8 \%$. Nevertheless, to prevent an oversimplification of the models we also investigated whether a double dipole-pair solution would yield better source estimates. Interestingly, this double dipole-pair solution did not appear superior to the single dipole-pair solution (see below).

\section{Results \\ N2 amplitudes}

In the baseline condition (adaptation to a static pattern), test motion patterns evoked a N2 at occipital electrodes for both high and low speeds. These slow and fast baseline N2 amplitudes were established for all subjects as the most negative amplitudes at one of the eight posterior electrodes within a latency window of 150-210 ms after stimulus onset. In order to establish modulation of the N2 amplitudes due to adaptation, the baseline N2 peak amplitudes needed to be large enough. For one subject the amplitude of the slow baseline N2 was above the acceptance criterion of $-2 \mu \mathrm{V}(-0.4 \mu \mathrm{V}$ for slow and $-2.1 \mu \mathrm{V}$ for fast). Data from this subject were excluded from further analysis. The average baseline peak amplitudes of the remaining 16 subjects were $-4.8 \mu \mathrm{V}$ (SEM $0.5 \mu \mathrm{V})$ at an average latency of $192 \mathrm{~ms}$ (SEM $3.6 \mathrm{~ms}$ ) for slow and $-8.6 \mu \mathrm{V}(\mathrm{SEM} 0.9 \mu \mathrm{V})$ at a latency of $190.4 \mathrm{~ms}$ (SEM $3.2 \mathrm{~ms}$ ) for fast motion.

The average evoked potential for both slow and fast baseline tests across 16 subjects (grand average VEPs) was calculated (Fig. 1). The N2 was visible at all posterior electrodes but was strongest at electrode POz. The response to fast motion was stronger than the response to slow motion at all electrodes. Interestingly, the VEPs did not fall back to baseline level after the $\mathrm{N} 2$ but remained negative and even showed another negative peak around $250 \mathrm{~ms}$ after stimulus onset. Peak N2 amplitudes for these grand average VEPs were $-2.1 \mu \mathrm{V}$ for the slow baseline and $-6.1 \mu \mathrm{V}$ for the fast baseline. The maximum $\mathrm{N} 2$ of both speeds occurred at electrode $\mathrm{POz}$ at a latency of $180 \mathrm{~ms}$ after stimulus onset.

For both fast and slow tests after both fast and slow adaptation, the grand average evoked potentials for motion in the same and opposite direction as the adaptation direction were calculated. At electrode $\mathrm{POz}$ (which had the strongest baseline N2), modulation of the N2 due to adaptation was clearly visible for conditions with congruent adaptation and test speed (Fig. 2, top left and bottom right panels). The results showed two different effects of adaptation. First, there was a global attenuation of VEPs, apparent for adaptation in both the opposite and same direction as the test direction when compared with the baseline VEPs. Second, the motion onset VEP that was adapted in the same direction as the test direction was much more strongly attenuated than the motion onset VEP that was adapted in the opposite direction, which means that modulation due to adaptation was partly direction selective.

In both conditions with incongruent adaptation and test speed (Fig. 2, top right and bottom left panels), adaptation effects were nearly absent. Only for fast tests following slow adaptation was the same direction-adapted N2 slightly attenuated compared with the baseline and opposite direction-adapted VEPs.

For 16 subjects, the N2 amplitudes for low and high test speeds after all adaptation conditions were established at the two electrodes (or single electrode if slow and fast motion responses for the baseline condition were maximal at the same electrode position) at which the individual slow and fast baseline $\mathrm{N} 2 \mathrm{~s}$ were at a maximum, in a latency window of $20 \mathrm{~ms}$ before to $20 \mathrm{~ms}$ after the latency of the baseline N2. 

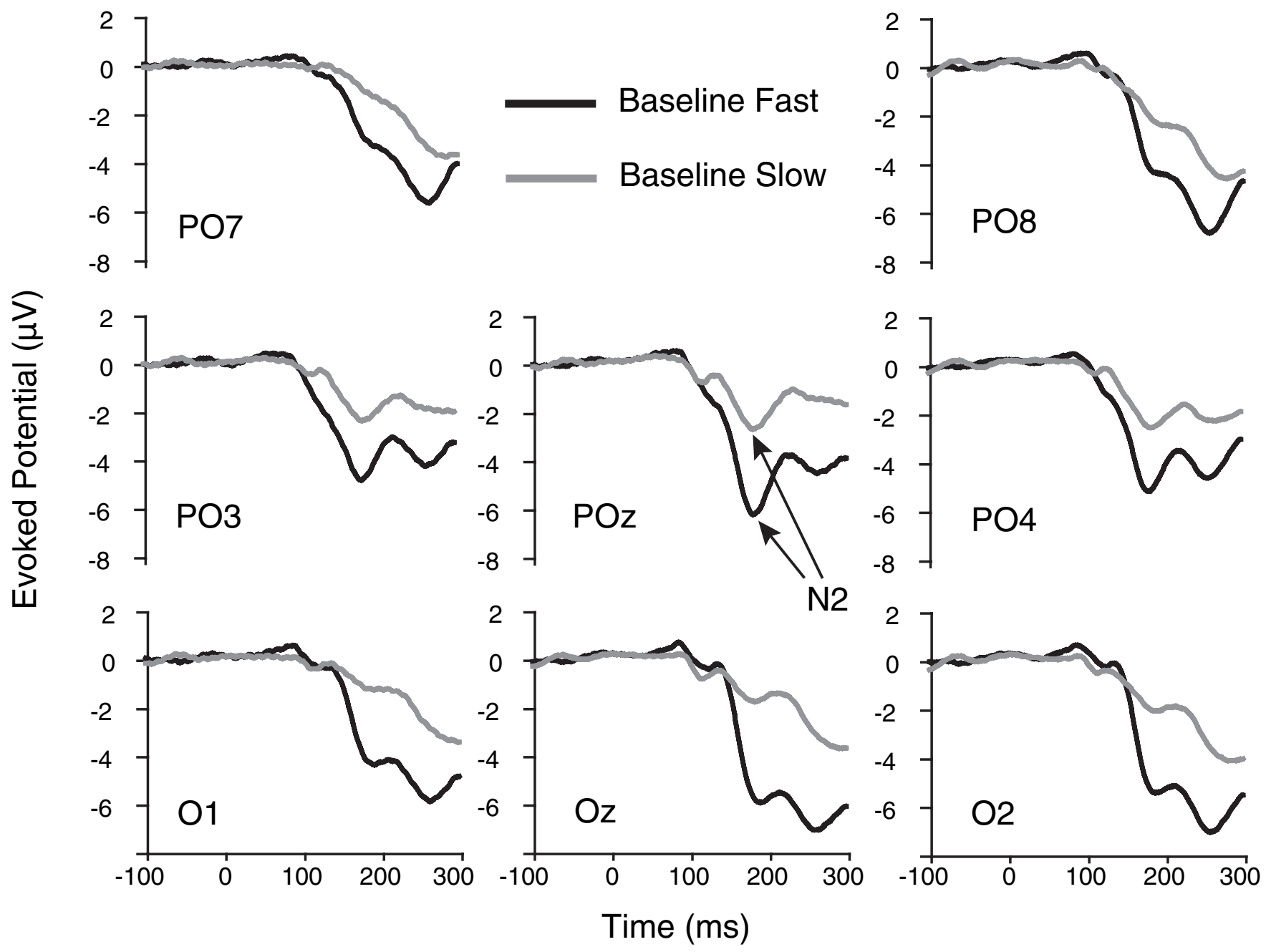

FIG. 1. The N2s within the grand average baseline evoked potentials (i.e. after adaptation to a stationary pattern) at eight posterior electrodes are stronger for fast test motion (black line) than for slow test motion (grey lines). For both speeds the N2 had maximum amplitude at electrode POz at a latency of 180 ms after test stimulus onset.

Individual N2 amplitudes after adaptation were normalized by dividing by the corresponding slow or fast baseline amplitudes. Thus, the modulation due to adaptation averaged over our population of subjects for slow and fast N2 amplitudes could be compared (Fig. 3)

First, adaptation resulted in a direction-independent amplitude reduction across most adaptation conditions compared with the baseline amplitudes. The N2 amplitudes of all adaptation conditions were compared with the corresponding fast or slow baseline in a paired $t$-test (Bonferroni corrected for multiple comparisons). Indeed, almost all adapted N2s were significantly reduced compared with the baseline N2 $(P<0.05$ for all comparisons), except for the fast test N2, which was preceded by a low adaptation speed in the opposite direction $(P=0.22)$.

Second, when the adapting and test motion had congruent speeds, differences in reduction between test motion in the same vs. opposite direction as the adaptation direction were found (Fig. 3, top left and bottom right panels). Paired $t$-tests showed that, for both slow and fast N2s adapted by congruent speeds, these differences were significant ( $P<0.0001$ for both comparisons).

In contrast to the data from Heinrich et al. (2004), the amplitude to high-speed onset after slow adaptation (incongruent speeds) was also significantly different between the same vs. opposite direction $(P=0.006$, Fig. 3, bottom left). However, this cross-speed adaptation was much weaker than the congruent-speed adaptations, as the N2 of the same direction was reduced by only $14 \%$ compared with the N2 of the opposite direction test. In contrast, N2 reduction was 59 and 44\% for slow and fast tests, respectively, after congruent speed adaptation. Only for the low-speed test after high-speed adaptation was there no significant difference in amplitude between directions $(P=0.100$, Fig. 3, top right).

In the previous analysis the electrode that was chosen to perform the calculations could be different for fast and slow tests. Therefore, we also compared N2 amplitudes for the same and opposite tests for a single electrode at which the baseline grand average N2 for both speeds was strongest (POz) and also for the average N2 across all eight posterior electrodes. Both comparisons showed the same significant differences between the same and opposite directions. Again, N2 amplitudes for both low and high speeds adapted by congruent speeds differed significantly $(P<0.0001$ for both comparisons), whereas the amplitude for the high speed after slow adaptation (incongruent speeds) was also significantly different between the same vs. opposite direction $(P=0.004)$. 


\section{Slow Adaptation}

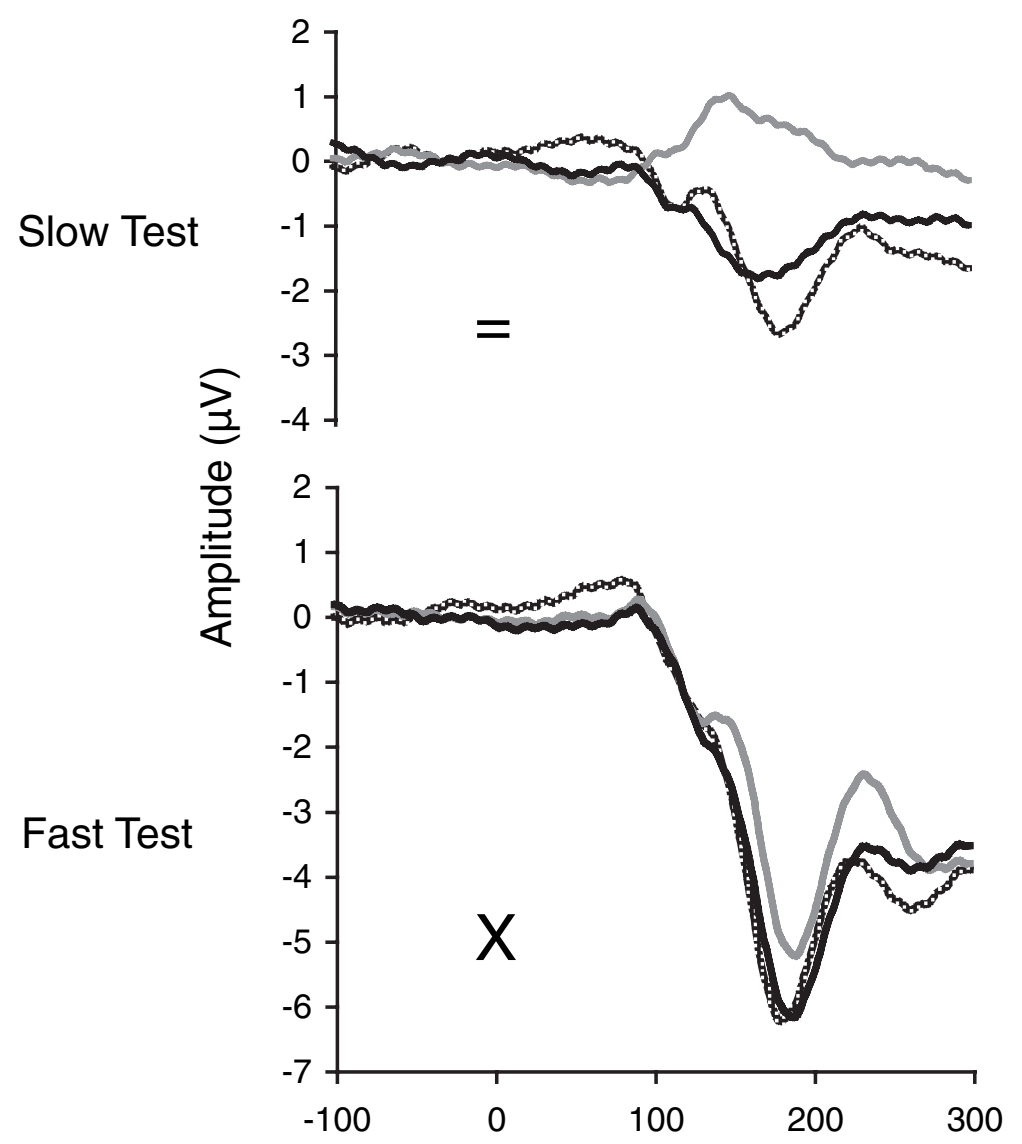

Fast Adaptation
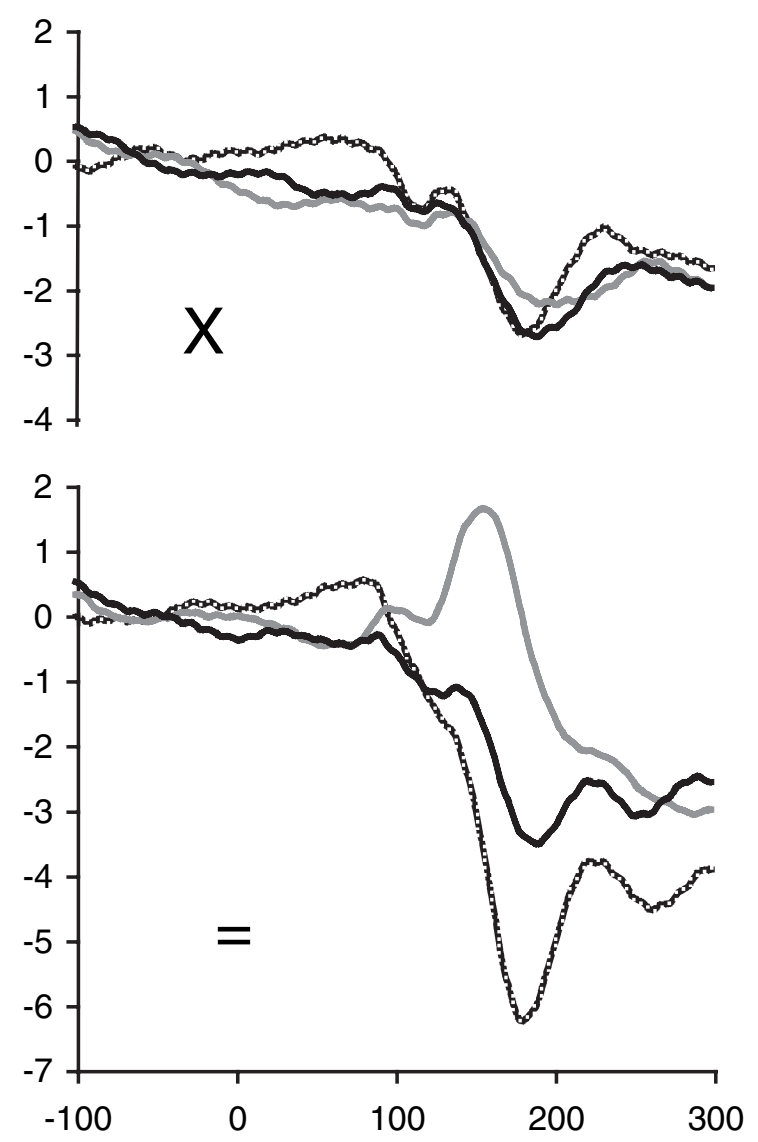

Time (ms)

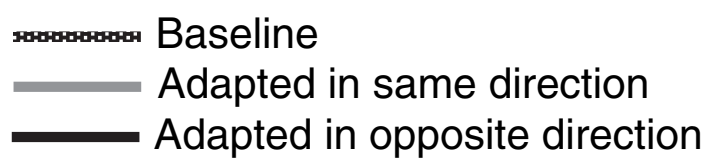

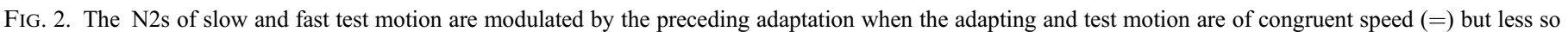

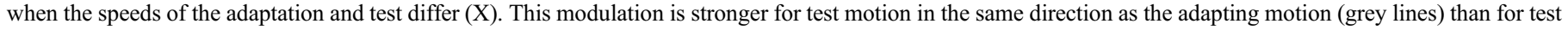
motion in the opposite direction as the adapting motion (black lines), compared with the baseline responses (patterned lines).

\section{Source analysis of baseline N2s}

For both unadapted baseline grand averages, the scalp distribution in a $3 \mathrm{~ms}$ window around the peak amplitude latency $(180 \mathrm{~ms}$ for both speeds) was established (Fig. 4). The response to fast motion was stronger than the response to slow motion. Further, the scalp distribution of the high-speed response had a much sharper gradient than the wider low-speed scalp potential distribution.

These scalp distributions were used for source modelling of the neuronal sources underlying the N2s. The data could be well fitted in a symmetrical dipole model. The RV was the percentage of the scalp data that could not be explained by the model. Therefore, the lower the $\mathrm{RV}$, the better the model fitted the data. The RV for the slow baseline source model was $2.44 \%$ and the RV for the fast baseline source model was $1.72 \%$, which means that both were excellent fits.

Source analysis revealed that the high-speed N2 arose from neural structures that were located posterior, dorsal and medial from the areas underlying the low-speed N2 (Fig. 4). To statistically test this trend, source analysis was performed on the individual N2 scalp distributions at the corresponding individual latencies. Gradients within the scalp data of two subjects were too weak to deliver valid source localization for the slow motion condition. Source locations of these subjects were excluded from statistical analysis that compared locations for fast and slow baseline N2s and those that compared locations of the slow baseline N2 with (slow) adapted sources. Individual $x, y$ and $z$ coordinates (see Fig. 4 for an explanation of these axes) for slow and fast motion onset were compared in a multivariate test (repeated-measures ANOVA) with speed (2) as within-subjects factor and coordinates (3) as measures. The difference in location for the baseline response of the two speeds was significant (for $N=14$, Hotelling Trace, $F_{3,11}=5.108$, $P=0.019)$. A univariate test revealed that this difference was not significant for any of the three axes separately (Greenhouse-Geisser corrected $P$-values, $0.055,0.067$ and 0.080 for $x, y$ and $z$ axes, 


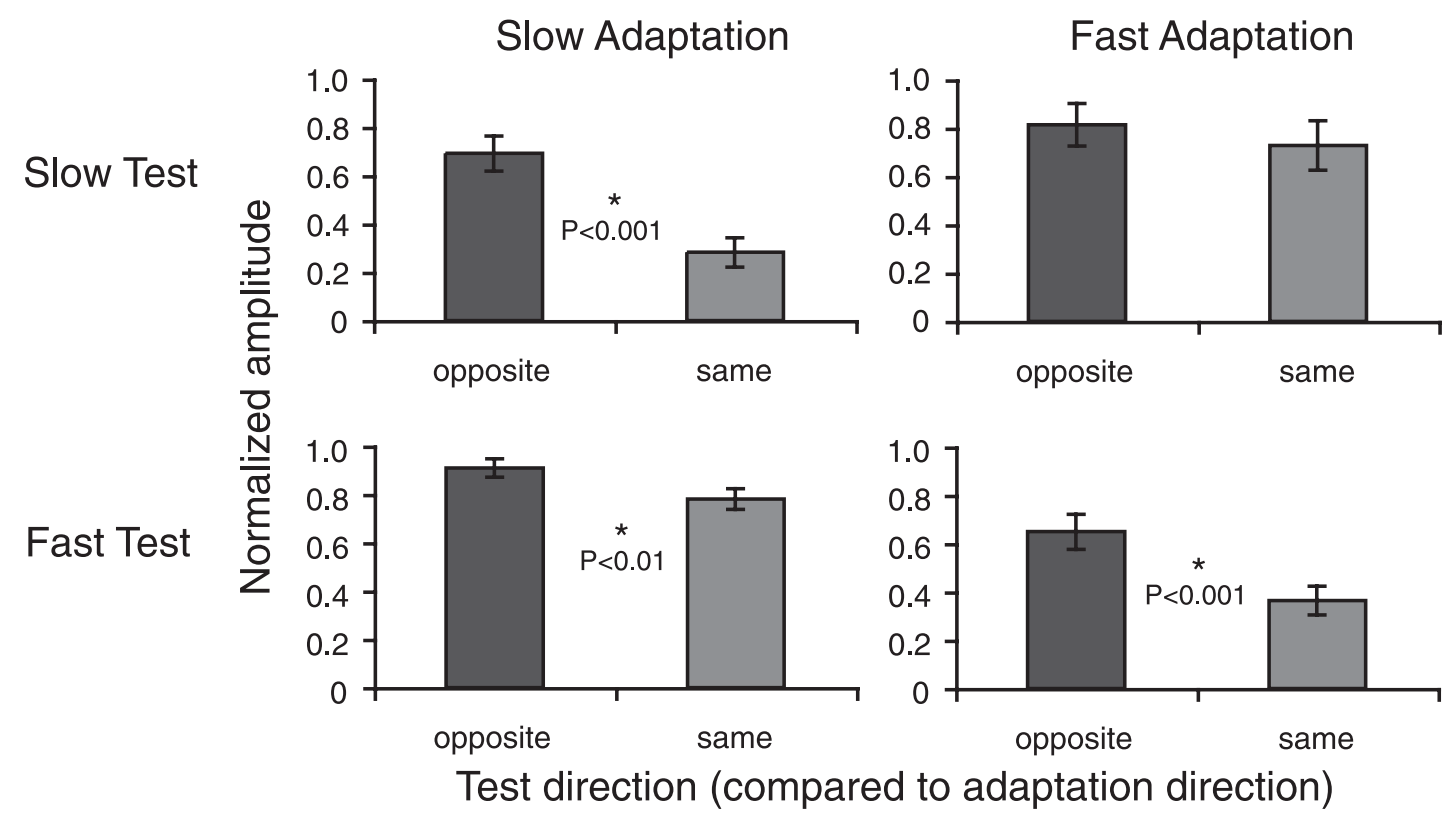

FIG. 3. The average normalized N2 amplitudes for low and high test speeds after adaptation at low or high adaptation speeds in the same or opposite direction as the preceding adaptation. Amplitudes were normalized for each subject by dividing through the corresponding slow or fast baseline N2 amplitude. *Significant differences in amplitude between opposite and same motion directions.

respectively), which means that the significant deviation between the two conditions should be attributed to a combination of differences along the three axes.

\section{Source analysis of N2 adaptation}

Although the differences in baseline N2 sources reflect different activated motion areas, they also reflect non-motion processing. To more specifically isolate motion processing, the VEP that was adapted by motion in the same direction was subtracted from its corresponding baseline VEP (i.e. with the same speed) for low and high test speeds (Fig. 5). Only tests with the same speed as the preceding adaptation were included in this subtraction and crossspeed adaptations were excluded. This difference reflected the maximum adaptation, encompassing both direction- and speedspecific components. Source locations of the grand average of these difference potentials showed the same trend as the baseline sources along the $x, y$ and $z$ axes, i.e. the high-speed $\mathrm{N} 2$ arose from neural structures that were located posterior and medial, and slightly dorsal, from the areas that processed the low-speed N2. The RV for the slow-source solution was $1.2 \%$ and the RV for the fast-source model was $0.8 \%$. Multivariate analysis on the individual sources revealed that locations were indeed significantly different $(N=16$, Hotelling Trace. $\left.F_{3,13}=6.462, P=0.007\right)$. Univariate analysis indicated that differences were significant along the $x$ axis (Greenhouse-Geisser $P=0.025)$ and along the $y$ axis (Greenhouse-Geisser $P=0.001$ ) but not along the $z$ axis (Greenhouse-Geisser $P=0.345$ ). The slight differences between this pattern of results and that for the baseline N2 suggests that the latter does reflect more than adaptable motiondependent responses.

\section{Direction-independent adaptation}

The baseline minus same subtraction reflects both direction-dependent and -independent motion-dependent responses, which could well originate from different areas, the geometrical average of which is estimated as the baseline minus same adapted source. To reduce the direction-specific contribution of the adaptation and to focus more on the speed-dependent part of the adapted N2, we subtracted the opposite-adapted N2 from the baseline N2. As directionally-dependent responses would be present in both $\mathrm{N} 2 \mathrm{~s}$, they would be strongly reduced in the subtraction N2. This N2 would then mainly reflect a non-directional adaptation component. Source localization revealed that these direction-independent components were even more strongly divided for responses to slow and fast motion (Fig. 6), and apparently also lateralized. The RV for the slow source model was $2.76 \%$ and the $\mathrm{RV}$ for the fast model was $7.59 \%$. Individual sources were again established to statistically quantify this difference. No reliable sources could be obtained for one subject in the fast condition and one subject in the slow condition. Therefore, these subjects were excluded from statistical comparisons regarding these sources. The sources of the remaining subjects were compared, again in multivariate and univariate tests, and revealed a significant difference in location between responses to slow and fast speeds (multivariate, $N=14$, Hotelling Trace, $\left.F_{3,11}=7.842, P=0.004\right)$. Locations differed along the $x$ and $y$ axes but not along the $z$ axis (Greenhouse-Geisser $P$-values, 0.013, 0.007 and 0.373 , respectively).

\section{Source analysis of direction-dependent N2 adaptation}

To investigate whether direction-selective processing occurs in different areas for low and high speeds, the difference potentials between tests in opposite vs. same direction as the adaptation were established for both low and high speeds (Fig. 7). These difference potentials again only included tests with the same speed as the preceding adaptation, thus excluding cross-speed adaptations. As this subtraction eliminated common components of the opposite- and same-adapted response, only the N2 components that were directionally adapted were reflected (as they are present in the opposite-adapted but not in the same-adapted response). 


\section{Slow Baseline}
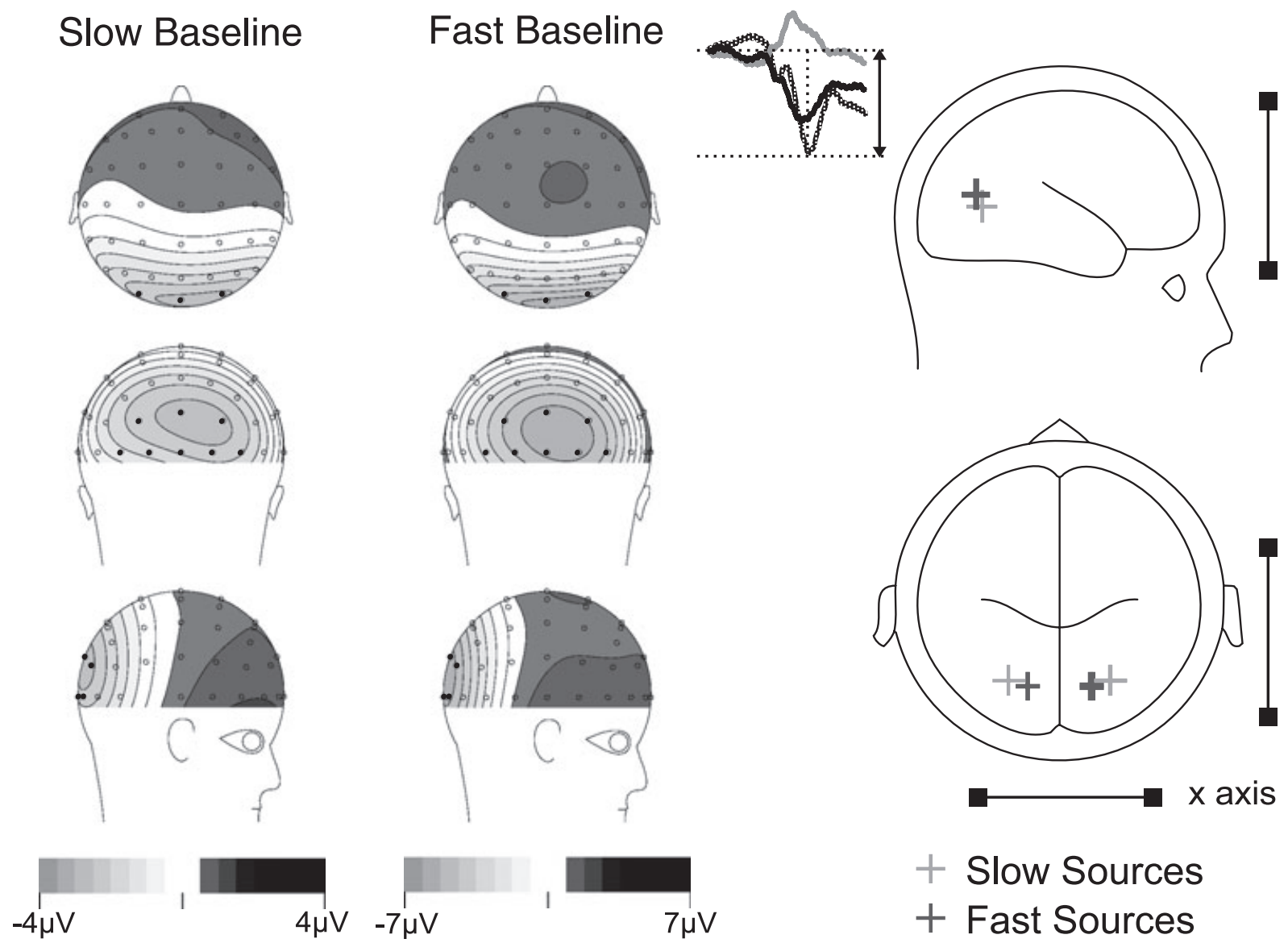

z axis
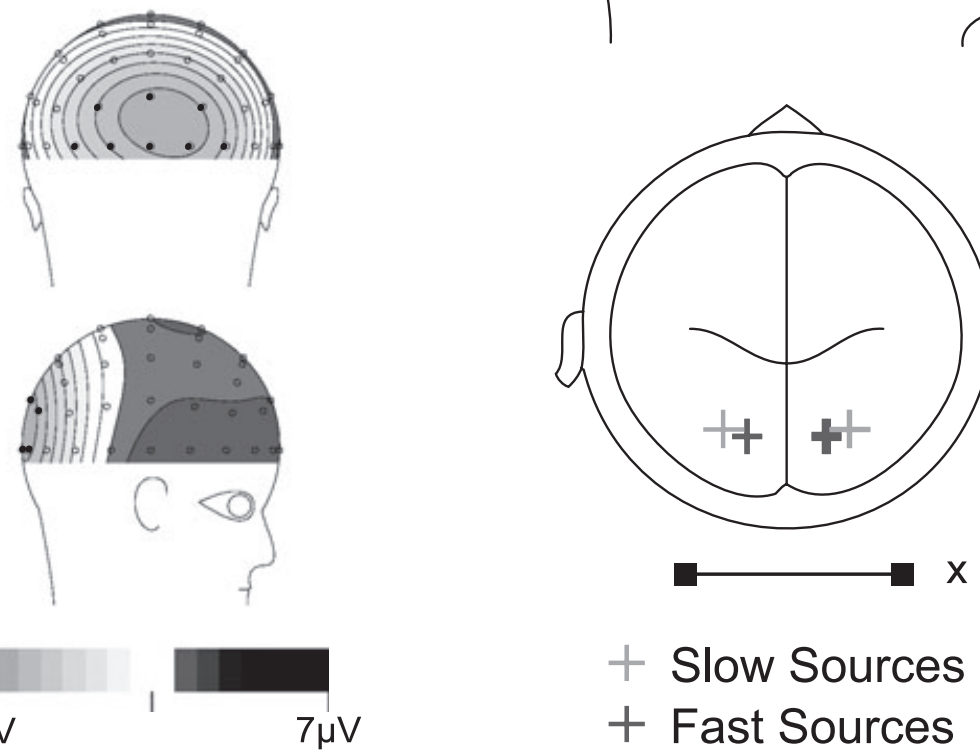

FIG. 4. The grand average scalp maps $(N=14)$ of the slow and fast baseline VEPs at $180 \mathrm{~ms}$ after stimulus onset are shown in the left and middle panel (see icon for the type of comparison; the icon does not depict the actual N2s for any particular electrode in this condition). Individual scalp distributions formed the basis for source localization of these baseline responses (right panel). The lines indicate the $95 \%$ certainty range of the individual source estimates in the $x, y$ and $z$ directions (dark grey, high speed; light grey, low speed). The relative strength of the dipoles is represented by the thickness of the lines. A slight right-hemisphere advantage as reported by Niedeggen \& Wist (1999) is apparent for the sources for both fast motion and, to a lesser extent, slow motion. Sources for fast motion were located more medial and slightly more posterior and dorsal than slow motion sources. The $x, y$ and $z$ axes are shown in the righthand panel. The eight posterior electrodes that were used in N2 amplitude analysis are marked with black dots in the scalp distribution maps.

Source localization of the grand average difference potential was performed for the same latency window as the baseline model $(180 \mathrm{~ms}$ for both speeds). BESA analysis revealed that directionally selective adaptation arose from the same or from closely located areas for both slow- and fast-speed processing. The RV for the slow source model was $2.8 \%$ and the RV for the fast model was $1.7 \%$. Multivariate analysis on the individual sources revealed that they did not differ in location for high or low speeds $(N=16$, Hotelling Trace, $\left.F_{3,13}=0.496, P=0.691\right)$. The combination of direction-dependentand direction-independent-adapted sources seems to be consistent with a geometrical average as reflected in the baseline minus same adapted estimated source.

Individual locations of the sources underlying the N2 directionspecific adaptations for low and high speeds were compared with the locations of the baseline $\mathrm{N} 2$ sources in multivariate and univariate tests. For low speeds, locations of baseline N2 activation and of N2 (direction-dependent) adaptation differed significantly $(N=14$ high speed, Hotelling Trace, $\left.F_{3,11}=12.33, P=0.001\right)$. Slow sources differed significantly along the $y$ and $z$ axes (Greenhouse-Geisser $P$-values $<0.001$ ) but not along the $x$ axis (Greenhouse-Geisser $P=0.571)$. For high speeds, locations of baseline N2 activation and direction-dependent $\mathrm{N} 2$ adaptation did not differ significantly (Hotelling Trace, $F_{3,11}=3.353, P=0.059$ ).

\section{Comparison of directionally-dependent and -independent N2 adaptation}

The direction-independent N2 adaptation revealed different sources for slow and fast motion processing but the direction-dependent $\mathrm{N} 2$ adaptation appeared to occur at the same location for both speeds. To test whether these two adaptation conditions were anatomically separate, we compared for both speeds the location of the direction-dependent vs. -independent N2 adaptation. For both high and low speeds, multivariate tests revealed that direction-dependent and -independent adaptation of the N2 occurred at different locations $(N=15$; fast: Hotelling Trace, $F_{3,12}=8.553, P=0.003$; slow: Hotelling Trace, $F_{3,12}=5.349$, $P=0.014)$. For high speed, this difference occurred mainly along the $z$ axis (Greenhouse-Geisser $P$-value $=0.002$ ) but also along the $x$ and $y$ axes (Greenhouse-Geisser $P$-values, 0.035 and 0.037 , respectively). For low speed this difference was only significant for the $z$ axis $(P$-value $=0.001)$ and not along the $x$ and $y$ axes (Greenhouse-Geisser $P$-values, 0.291 and 0.204 , respectively).

\section{Single vs. multiple dipole pairs}

As it is possible that more than a single area is involved in (lowand/or high-speed) motion processing, it could be argued that our 

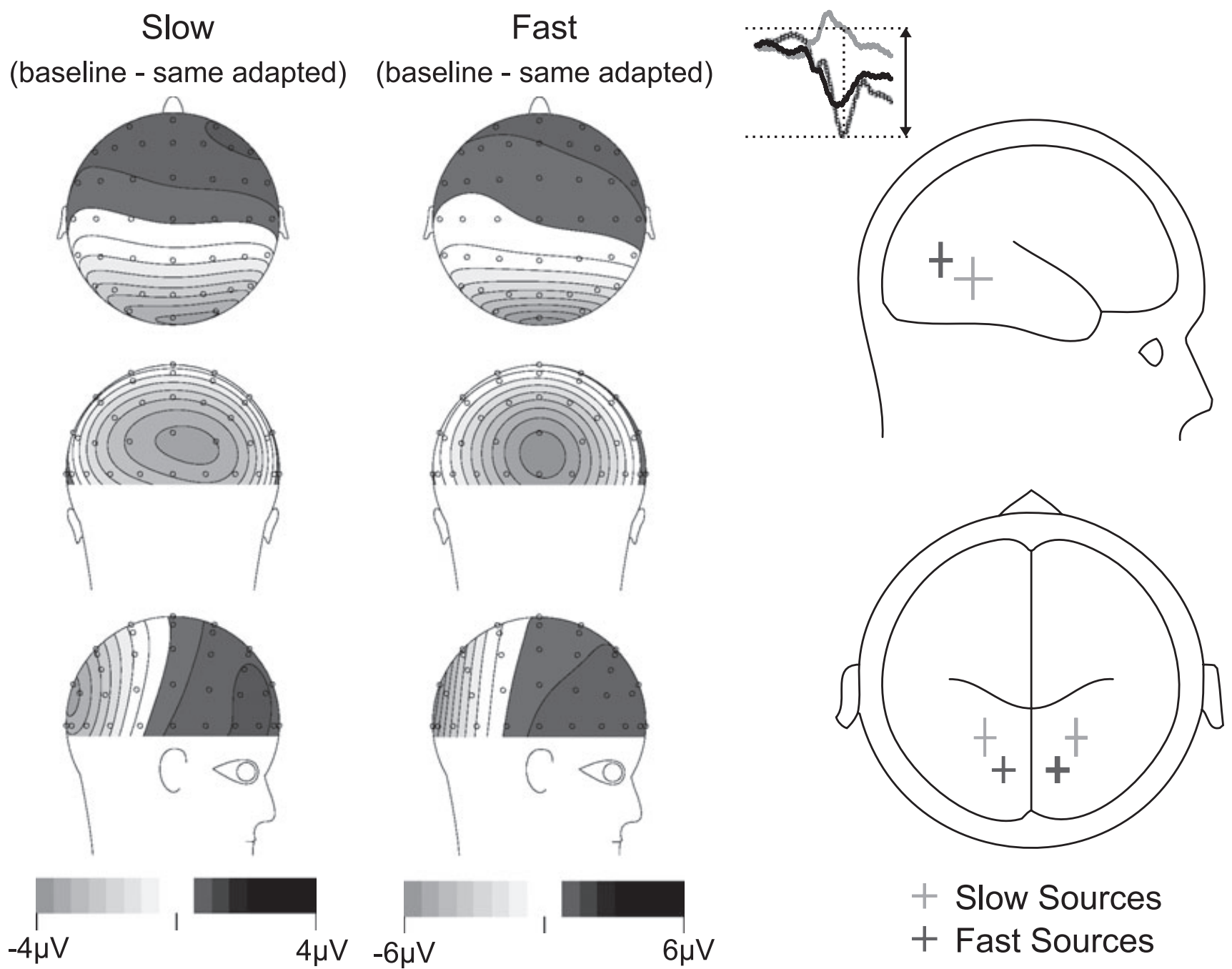

FIG. 5. Grand average scalp maps and source localizations for baseline VEPs - VEPs adapted in the same direction (see icon for comparison) identified the cortical regions underlying the maximum adaptation for low and high speeds $(N=16)$. Differences along the $x$ and $y$ axes were statistically significant. (Same conventions as Fig. 4.) As in Fig. 4, sources appear slightly stronger in the right hemisphere.

bilaterally constrained single dipole-pair model may have yielded oversimplified results. To address this possible confound, we reanalysed our comparisons with a bilaterally constrained double dipole-pair model. As can be expected when the degrees of freedom in a model are increased, the RV of the model decreased (compared with the single dipole-pair model). However, the additional dipoles were mostly frontal (not a likely location for motion processing) and much lower in strength than the original dipole solutions. Most importantly, the original sources from the two-dipole model did not differ significantly in location from their remodelled locations in the fourdipole model $(F<2.777, P>0.083$ for all comparisons; $F<1.888$, $P>0.19$ for all but the former).

\section{Discussion}

\section{Direction- and speed-specific N2 adaptation}

The EEG potentials evoked by the onset of slow- and fast-moving RPAs were recorded. These test RPAs were preceded by an adaptation period during which an RPA was presented that could (i) remain static for the unadapted baseline conditions, (ii) move at high speed $\left(32^{\circ} / \mathrm{s}\right)$ and (iii) move at low speed $\left(3.5^{\circ} / \mathrm{s}\right)$. The moving RPAs could move in the same or opposite direction as the test RPA. The two speeds were chosen based on psychophysical studies of the MAE, according to which motion at these speeds is processed, respectively, by strictly high temporal frequency channels and mainly low temporal frequency channels (Verstraten et al., 1998, 1999; van der Smagt et al., 1999). VEPs contained a typical negative peak between 150 and $200 \mathrm{~ms}$, which was named the N2. Amplitudes and source estimates of N2s evoked by slow and fast motion after different adaptation conditions were compared.

The N2 was followed by an extra negative peak at approximately $250 \mathrm{~ms}$ after stimulus onset. This peak may correspond to a parietooccipital negativity that occurs around $300 \mathrm{~ms}$ after stimulus onset and has been shown to represent subjective perception of visual motion perception, irrespective of the physical properties of the stimulus (Haarmeier \& Thier, 1998). In contrast to the current study, this peak was much less pronounced in the results of Heinrich et al. (2004), even though visual stimulation and instructions in both studies were closely matched. Here, however, we focus on modulation of the N2 by motion adaptation.

The amplitude of the N2 was modulated by the preceding adaptation, which indicates that this peak mainly reflects motion 

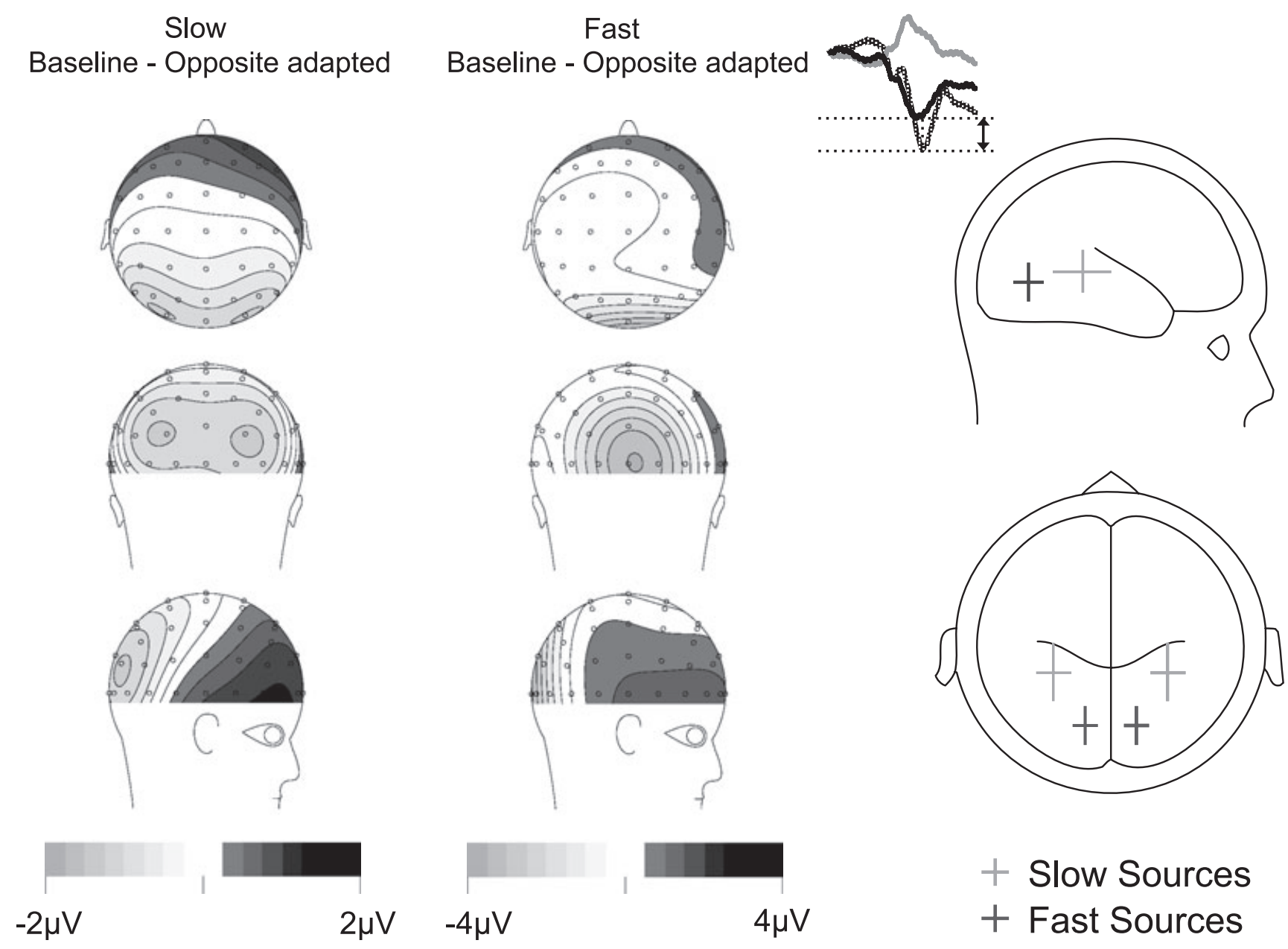

FIG. 6. Grand average scalp maps and source localizations for baseline VEPs - VEPs adapted in the opposite direction (see icon for comparison) revealed significant differences in neural structures for direction-independent adaptation $(N=14)$. Adapted regions for the N2 evoked by low speeds were significantly more anterior and lateral compared with adapted regions for N2s evoked by high speeds. (Same conventions as Fig. 4.)

processing, as has been shown in several studies (Bach \& Ullrich, 1994; Niedeggen \& Wist, 1998; Hoffmann et al., 2001; Heinrich et al., 2004; Muller et al., 2004; Lorteije et al., 2006, 2007). In concurrence with the results from Heinrich et al. (2004), modulation of the test $\mathrm{N} 2$ depended on both the speed and direction of the adapting motion. However, in addition to the direction-specific adaptation for conditions with congruent adaptation and test speed, also found by Heinrich et al. (2004), a much smaller but significant direction-specific adaptation was found for high speeds that were preceded by a low-speed adaptation. Only low speeds that were preceded by high-speed adaptation did not contain this directionspecific adaptation.

Thus, these results corroborate in part the findings of Heinrich et al. (2004) of separate processing of slow and fast motion. The additional direction-specific attenuation of the fast motion response by slow motion adaptation revealed that the speed ranges of the two channels are not completely distinct. Instead, the high-speed evoked $\mathrm{N} 2$ arose from direction-selective neural structures that were also involved in direction-selective processing of slow motion. In contrast, the low-speed N2 arose mainly from direction-selective neurones that were not involved in direction-selective processing of fast motion. This result is in accordance with psychophysical data, which have revealed that the suggested low-speed motion channel has a tuning range for speeds below $\sim 25^{\circ} / \mathrm{s}$ and peaking around $3 \%$, whereas the suggested high-speed motion channel has a higher range from below $1 \%$ s up to $80^{\circ} / \mathrm{s}$ (Verstraten et al., 1998; van de Grind et al., 2001; Curran \& Benton, 2006). Due to this overlap, a single intermediate speed can evoke a transparent MAE on a test pattern containing both slow and fast randomly moving dots (Curran \& Benton, 2006).

In other words, the high speed used in the current experiments fell well outside the speed-tuning range of the low-speed channel, whereas the low speed used still fell within the (much broader) speed-tuning range of the high-speed channel and thus caused a certain degree of adaptation. However, this cross-speed N2 reduction is much smaller in amplitude than the congruent-speed N2 reductions. Thus, it may have been insignificant in the study of Heinrich et al. (2004), whereas the congruent reductions found in that study did reach significance. The current study used more electrodes for a more optimal N2 recording and a larger number of participants, which probably yielded sufficient statistical power to detect this smaller reduction.

It should be noted that the baseline condition in the present experiment may not be completely unadapted, as here adaptation was to a static pattern that also adapts motion channels (e.g. van de Grind et al., 2004; van Boxtel et al., 2006). This especially holds for the low speeds, for which the baseline N2 amplitude was indeed lower than 

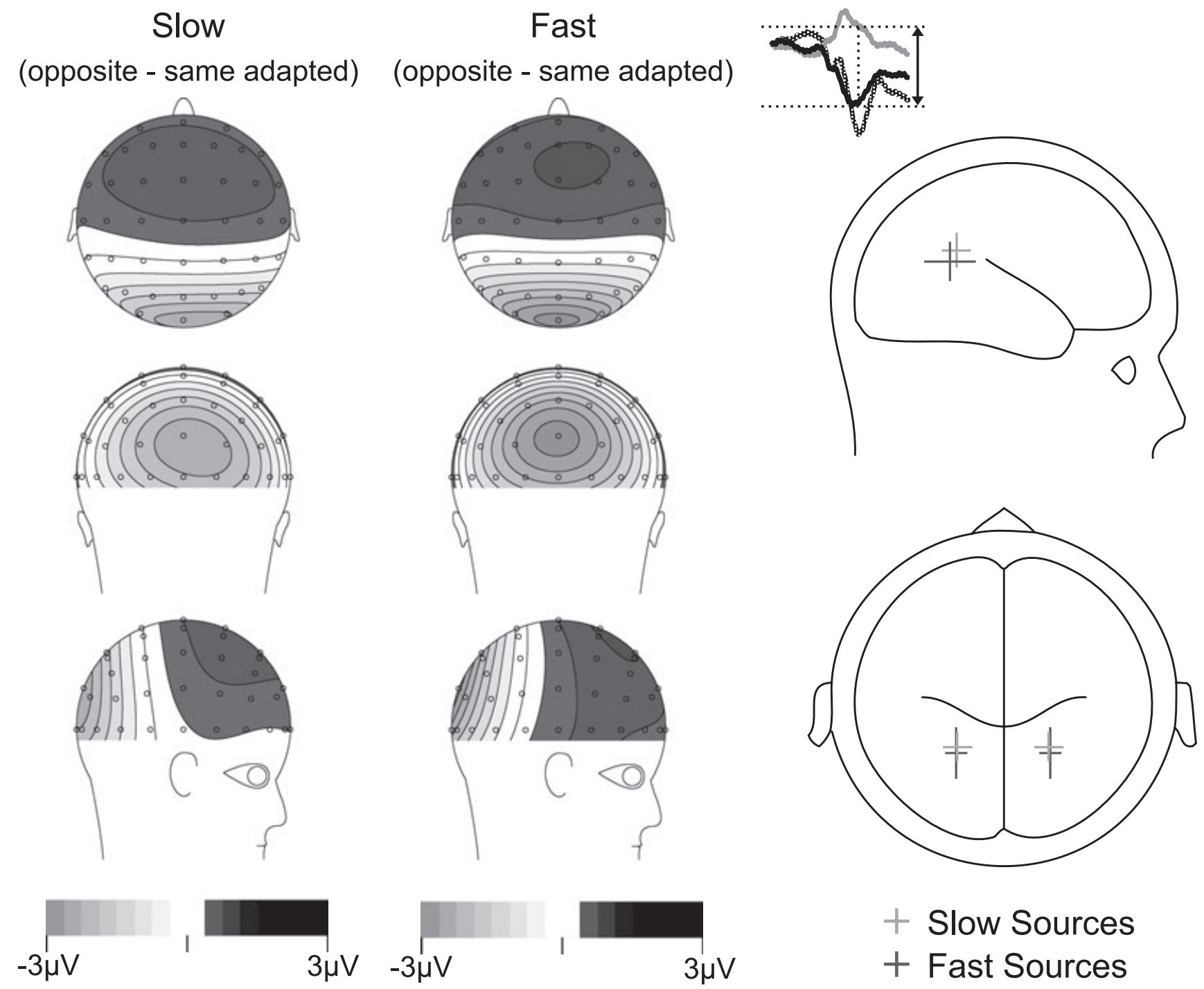

FIG. 7. Grand average scalp maps and source localizations for VEPs adapted in the opposite direction - VEPs adapted in the same direction (see icon for comparison) revealed no differences in neural structures for direction-dependent adaptation $(N=16)$. (Same conventions as Fig. 4.)

that for the high speed used. This might also have implications for the size of the direction-independent adaptation effect but should not affect the size of the direction-dependent adaptation or the source localizations of any condition.

\section{Different cortical areas are involved in processing of low and high speeds}

Source localization of the baseline responses to fast and slow motion revealed that their corresponding N2s arose from different areas (see Table 2 for a summary of the comparisons for all conditions). Sources contributing to the N2 response to slow motion onset were found more anterior, lateral and slightly more ventral than sources underlying the N2 response to fast motion onset (see Fig. 4). [It is important to note that, rather than identifying exact anatomical regions, BESA can deliver relative locations of neuronal sources. Source locations of N2 responses to low- and high-speed motion (and their adaptation) can thus be compared but without reference to specific cortical areas.]
The N2 response can be divided into three sub-components: a nonadaptable contributor and two, probably distinct, adaptable contributors, one independent of adaptation direction and one depending on adaptation direction (e.g. Hoffmann et al., 2001; Heinrich et al., 2004). Subtraction of the maximally adapted potentials (same speed and same direction) from baseline potentials eliminates responses from areas that were not adapted, leaving only response areas adapted by motion. These areas differed for low and high speeds along the $x$ and $y$ axes, and showed nearly the same pattern as the baseline sources; fast motion adaptation occurred more medial and posterior than slow motion adaptation, only the dorsal/ventral divergence was not significant (see Fig. 5).

Having established that areas that were adapted by our motion stimulus differed in location for low and high speeds, we further analysed whether direction-independent or -dependent adaptation (or both) gave rise to this source location difference. Our results show that direction-independent adaptation appears to be the main contributor to the difference in location of the sources underlying the motion onset VEP (N2 component). Sources for direction-independent N2 
TABLE 2. Summary of dipole source localizations and comparisons

\begin{tabular}{|c|c|c|c|c|c|c|c|c|}
\hline \multirow[b]{2}{*}{ Condition } & \multirow[b]{2}{*}{ Comparison } & \multicolumn{3}{|l|}{ Slow } & \multicolumn{3}{|l|}{ Fast } & \multirow{2}{*}{$\begin{array}{l}\text { Significant } \\
\text { difference }\end{array}$} \\
\hline & & $x$ & $y$ & $z$ & $x$ & $y$ & $z$ & \\
\hline Baseline & Baseline N2 & -0.3 & -0.5 & 0 & -0.2 & -0.5 & 0.2 & $*$ \\
\hline Full adaptation & Baseline N2 - same adaptation & -0.3 & -0.3 & 0.1 & -0.2 & -0.5 & 0.2 & $*$ \\
\hline Direction-independent adaptation & Baseline N2 - opposite adaptation & -0.4 & -0.2 & 0.1 & -0.2 & -0.5 & 0 & $*$ \\
\hline \multirow[t]{3}{*}{ Direction-dependent adaptation } & Opposite adapt - same adaptation & -0.3 & -0.3 & 0.3 & -0.3 & -0.3 & 0.3 & - \\
\hline & Baseline vs. direction-dependent & - & $*$ & * & - & - & - & \\
\hline & Direction-independent vs. direction-dependent & - & - & * & $*$ & $*$ & $*$ & \\
\hline
\end{tabular}

The $x, y$ and $z$ coordinates are from BESA analyses. *Significant difference between source locations (multivariate or univariate along at least one dimension).

adaptation for slow test motion were significantly more anterior and lateral compared with sources for fast test motion (see Fig. 6).

Sources for direction-dependent N2 adaptation to low and high speeds, however, did not appear to originate from different locations (see Fig. 7). This might be partly attributed to the low spatial resolution of VEP source modelling and the fact that the directionspecific N2 reduction is a much weaker signal than, for instance, the unsubtracted baseline $\mathrm{N} 2$ as fewer neurones are represented in this signal. However, spatial resolution was high enough to discriminate between these sources of direction-dependent adaptation and the sources of the direction-independent adaptation, which indicates that the spatial resolution and signal strength are indeed strong enough for reliable source estimates and that at least one area processes motion direction at both low and high speeds.

The N2 amplitude reduction due to adaptation already revealed that speed tuning of the fast motion channel was broad and overlapped with tuning of the slow motion channel. Therefore, it could be argued that the fast motion channel contributes to both fast- and (perhaps to a lesser extent) slow-adapted N2s, thus 'pulling' the source estimates for both speeds together. Moreover, these sources were obtained from the difference potential of the opposite- and same-adapted N2s, and, although this difference is similar in strength, the absolute response from this area to low and high speeds may differ.

\section{Motion vs. flicker adaptation}

It might be argued that the different areas underlying directionindependent fast and slow adaptation are not areas sensitive to motion information per se but rather areas that respond to flicker (e.g. Hoffmann et al., 2001; Maurer \& Bach, 2003; Heinrich, 2007). However, we propose a different interpretation, at least for our stimuli. The dense RPAs used in our experiments contain many spurious motion signals from frame to frame. A single shift of all pixels in such an RPA will cause a large amount of motion energy in the direction of the shift and will thus excite a large number of motion sensors tuned to that direction/speed combination. However, many more direction/speed combinations are present in such a two-frame display, due to all the different possible correspondences. In a prolonged exposure to such a display, many local motion sensors will be excited and, to a certain extent, adapted. However, this adaptation will be less strong and for all directions (and speeds). It is only at the stage where a strict motion opponency kicks in [presumably at or above the middle temporal region (MT)] or in regions with cells with much larger receptive fields (or more tuned to global motion) that this local motion adaptation will have lost its influence.

Moreover, the frame-to-frame refresh frequency of our stimuli was identical in the low- and high-speed conditions, as were the broadband spatial characteristics of each frame. The space/time relationship, i.e. the step size of the coherent displacement of the pixels, is the only physical difference between the slow and fast stimuli in our experiment. If the opposite-direction condition would only adapt a 'flicker' mechanism, low- and high-speed oppositedirection stimulation should result in adaptation of exactly the same neural substrate. Hence, a flicker mechanism cannot explain the different source localizations for direction-independent adaptation shown in Fig. 6.

\section{N2 source locations}

The relative locations of dipole sources for slow- and fast-motion N2s are very interesting, as the anterior/posterior difference is opposite to what could be expected based on extracellular recordings in macaques, which revealed that neurones in the posterior primary visual area detect directional differences at lower speeds than neurones in the relatively more anterior MT (e.g. Mikami et al., 1986; Churchland et al., 2005). Additionally, the macaque area MT shows a stronger functional MRI (fMRI) BOLD response when presented with fast motion $\left(8^{\circ} / \mathrm{s}\right)$ than when presented with slow motion $\left(4^{\circ} / \mathrm{s}\right)$ (Nelissen et al., 2006). In humans, BOLD responses in visual areas have also been compared. Chawla et al. $(1998,1999)$ have shown increased BOLD activation (compared with stationary stimuli) in all areas tested for all speeds used (up to $\sim 60^{\circ} / \mathrm{s}$ ). Activity in the primary visual area was shown to decrease with increasing stimulus speed, whereas for instance area V3a and MT appeared tuned to intermediate speeds (MT tuned to slightly higher speeds than V3a). The speed ranges reported are variable and appear to differ between the two studies (from between $7 \% \mathrm{~s}$ and $30 \% \mathrm{~s}$ to between $4 \% \mathrm{~s}$ and $16^{\circ} / \mathrm{s}$ in the latter study). As the fMRI data comparing speed tuning in different brain areas are sparse and show such variable outcomes, it would be useful to implement an fMRI-adaptation paradigm to complement the results reported here.

\section{Disentangling direction-dependent and -independent motion processing for both speeds}

For N2s in response to both slow and fast motion, source locations for direction-independent adaptation were significantly more ventral compared with source locations for direction-dependent adaptation. This is of interest as the cortical visual system is generally assumed to be divided into two major pathways: a more dorsal visual stream, often referred to as the 'where' pathway, and a more ventral visual stream, often referred to as the 'what' pathway (e.g. Ungerleider \& Mishkin, 1982). The transient response characteristics of the dorsal pathway vs. the sustained response characteristics of the ventral pathway suggest that this division across the two cortical streams may correspond to a 
division in earlier stages of visual processing that are divided across, respectively, a magnocellular and a parvocellular route (e.g. DeYoe \& Van Essen, 1988). The transient vs. sustained response characteristics indicate that this division may underlie the separate high- and lowspeed channels. This notion might explain various psychophysical results on the static and dynamic MAEs (Verstraten et al., 1998; van der Smagt et al., 1999), different binocular rivalry stages for low- and high-speed motion (van de Grind et al., 2001), and interaction between colour and motion processing (Gegenfurtner \& Hawken, 1996).

The present data, however, do not support such a simple slow-toventral and fast-to-dorsal mapping, as direction-dependent adaptation was found in more dorsal areas for both low and high speeds. Direction-independent adaptation still allows for slow-to-ventral and fast-to-dorsal mapping, and this adaptation may be qualitatively different from direction-dependent adaptation. Our findings thus suggest a division of motion processing along a more ventral direction-independent pathway for low speeds and a more dorsal direction-dependent pathway for both high and low speeds. As N2 amplitudes were modulated in a combined speed-and-directiondependent manner, this reveals that more dorsal visual areas may process both low and high speeds but that this process occurs separately within different neural populations. Based on the dorsal location of these sources, they may represent dorsal motion areas such as area MT.

It has been postulated that the dorsal high-frequency channel processes motion information, whereas the ventral low-frequency channel is involved in pattern and shape processing (Kulikowski, 1971; Georgeson, 1985; van de Grind et al., 2001). This idea corresponded to work by Probst et al. (1993), who showed that both motion onset and pattern reversal (which is a directionindependent 'motion' stimulus) evoked an N2 peak. Sources for pattern onset were more anterior and medial compared with sources for motion onset. In addition, different sources for different types of motion (e.g. translation vs. rotation) have recently been demonstrated (e.g. Wang et al., 1999; Delon-Martin et al., 2006). Source activity due to motion onset as recorded with magnetoencephalograms also reveals temporal occipital sources but with the most prominent peaks occurring between 200 and $300 \mathrm{~ms}$ (Amano et al., 2006), which is later than the $\mathrm{N} 2$ peak as recorded with EEG.

As has been suggested by van de Grind et al. (2001), a ventral motion pathway may be vital for obtaining structure from motion. In addition, it may correspond to the dynamic form pathway as postulated by Zeki (1993), for which area V3 has been proposed to be an important processing stage (Zeki, 1993; Gegenfurtner et al., 1997). Support for this notion comes from a study by Wang et al. (1999), who demonstrated strong dipole sources in ventral areas in response to form-from-motion stimuli and much weaker dipole sources in response to pure motion stimuli, whereas dorsal dipole sources responded strongly to both.

Even though direction-dependent adaptation mainly affected the N2 components arising from the dorsal visual pathway, this does not necessarily rule out any direction sensitivity in the ventral stream. Direction sensitivity may be much stronger in the dorsal stream, thus 'pulling' the direction-dependent dipole sources toward the dorsal stream.

In conclusion, we have shown, for the first time, physiological evidence that motion with low speed is processed along the ventral pathway, whereas the dorsal motion-processing pathway has a much larger range of speed tuning and is divided into two neural (sub-)populations.

\section{Acknowledgements}

We thank Wim van de Grind and Michael Hoffmann for valuable comments on earlier drafts of this manuscript. This research was supported by the Netherlands Organization for Scientific Research (NWO).

\section{Abbreviations}

BESA, brain electric source analysis; EEG, electroencephalography; MAE, motion aftereffect; MT, middle temporal region; RPA, random pixel array; RV, residual variance; VEP, visually evoked potential.

\section{References}

Amano, K., Nishida, S. \& Takeda, T. (2006) MEG responses correlated with the visual perception of velocity change. Vis. Res., 46, 336-345.

Anderson, S.J. \& Burr, D.C. (1985) Spatial and temporal selectivity of the human motion detection system. Vis. Res., 25, 1147-1154.

Andreassi, J.L. \& Juszczak, N.M. (1982) Hemispheric sex differences in response to apparently moving stimuli as indicated by visual evoked potentials. Int. J. Neurosci., 17, 83-91.

Bach, M. \& Hoffmann, M.B. (2000) Visual motion detection in man is governed by non-retinal mechanisms. Vis. Res., 40, 2379-2385.

Bach, M. \& Ullrich, D. (1994) Motion adaptation governs the shape of motionevoked cortical potentials. Vis. Res., 34, 1541-1547.

Berg, P. \& Scherg, M. (1994) BESA Version 2.0 Handbook. Megis. Munich.

van Boxtel, J.J., van Ee, R. \& Erkelens, C.J. (2006) A single system explains human speed perception. J. Cogn. Neurosci., 18, 1808-1819.

Buchner, H., Gobbele, R., Wagner, M., Fuchs, M., Waberski, T.D. \& Beckmann, R. (1997) Fast visual evoked potential input into human area V5. Neuroreport, 8, 2419-2422.

Chawla, D., Phillips, J., Buechel, C., Edwards, R. \& Friston, K.J. (1998) Speeddependent motion-sensitive responses in V5: an fMRI study. Neuroimage, 7, 86-96.

Chawla, D., Buechel, C., Edwards, R., Howseman, A., Josephs, O., Ashburner, J. \& Friston, K.J. (1999) Speed-dependent responses in V5: a replication study. Neuroimage, 9, 508-515.

Churchland, M.M., Priebe, N.J. \& Lisberger, S.G. (2005) Comparison of the spatial limits on direction selectivity in visual areas MT and V1. J. Neurophysiol., 93, 1235-1245.

Curran, W. \& Benton, C.P. (2006) Test stimulus characteristics determine the perceived speed of the dynamic motion aftereffect. Vis. Res., 46, 32843290.

Delon-Martin, C., Gobbele, R., Buchner, H., Haug, B.A., Antal, A., Darvas, F. \& Paulus, W. (2006) Temporal pattern of source activities evoked by different types of motion onset stimuli. Neuroimage, 31, 1567-1579.

DeYoe, E.A. \& Van Essen, D.C. (1988) Concurrent processing streams in monkey visual cortex. Trends Neurosci., 11, 219-226.

ffytche, D.H., Guy, C.N. \& Zeki, S. (1995) The parallel visual motion inputs into areas V1 and V5 of human cerebral cortex. Brain, 118 (6), 13751394.

ffytche, D.H., Howseman, A., Edwards, R., Sandeman, D.R. \& Zeki, S. (2000) Human area V5 and motion in the ipsilateral visual field. Eur. J. Neurosci., 12, 3015-3025.

Gegenfurtner, K.R. \& Hawken, M.J. (1996) Interaction of motion and color in the visual pathways. Trends Neurosci., 19, 394-401.

Gegenfurtner, K.R., Kiper, D.C. \& Levitt, J.B. (1997) Functional properties of neurons in macaque area V3. J. Neurophysiol., 77, 1906-1923.

Georgeson, M.A. (1985) Inferring cortical organization from subjective visual patterns. In Rose, D. and Dobson, V.G. (Eds), Models of the Visual Cortex. John Wiley \& Sons Ltd., New York, NY, pp. 223-232.

van de Grind, W.A., van Hof, P., van der Smagt, M.J. \& Verstraten, F.A. (2001) Slow and fast visual motion channels have independent binocular-rivalry stages. Proc. Biol. Sci., 268, 437-443.

van de Grind, W.A., van der Smagt, M.J. \& Verstraten, F.A.J. (2004) Storage for free: a surprising property of a simple gain-control model of motion aftereffects. Vis. Res., 44, 2269-2284.

Haarmeier, T. \& Thier, P. (1998) An electrophysiological correlate of visual motion awareness in man. J. Cogn. Neurosci., 10, 464-471.

Heinrich, S.P. (2007) A primer on motion visual evoked potentials. Doc. Ophthalmol., 114, 83-105.

Heinrich, S.P. \& Bach, M. (2003) Adaptation characteristics of steady-state motion visual evoked potentials. Clin. Neurophysiol., 114, 1359-1366. 
Heinrich, S.P., van der Smagt, M.J., Bach, M. \& Hoffmann, M.B. (2004) Electrophysiological evidence for independent speed channels in human motion processing. J. Vis., 4, 469-475.

Hoffmann, M.B., Dorn, T.J. \& Bach, M. (1999) Time course of motion adaptation: motion-onset visual evoked potentials and subjective estimates. Vis. Res., 39, 437-444.

Hoffmann, M.B., Unsold, A.S. \& Bach, M. (2001) Directional tuning of human motion adaptation as reflected by the motion VEP. Vis. Res., 41, 2187-2194.

Kubova, Z., Kuba, M., Spekreijse, H. \& Blakemore, C. (1995) Contrast dependence of motion-onset and pattern-reversal evoked potentials. Vis. Res., 35, 197-205.

Kulikowski, J.J. (1971) Effect of eye movements on the contrast sensitivity of spatio-temporal patterns. Vis. Res., 11, 261-273.

Kulikowski, J.J. \& Tolhurst, D.J. (1973) Psychophysical evidence for sustained and transient detectors in human vision. J. Physiol., 232, 149-162.

Lorteije, J.A.M., Kenemans, J.L., Jellema, T., van der Lubbe, R.H.J., de Heer, F. \& van Wezel, R.J.A. (2006) Delayed response to animate implied motion in human motion processing areas. J. Cogn. Neurosci., 18, 158-168.

Lorteije, J.A.M., Kenemans, J.L., Jellema, T., van der Lubbe, R.H.J., Lommers, M.W. \& van Wezel, R.J.A. (2007) Adaptation to real motion reveals direction selective interactions between real and implied motion processing. J. Cogn. Neurosci., 19, 1231-1240.

Mather, G., Verstraten, F.A.J. \& Anstis, S.M. (1998) The Motion Aftereffect: A Modern Perspective. MIT Press, Cambridge, MA.

Maurer, J.P. \& Bach, M. (2003) Isolating motion responses in visual evoked potentials by preadapting flicker-sensitive mechanisms. Exp. Brain Res., 151, 536-541.

Mikami, A., Newsome, W.T. \& Wurtz, R.H. (1986) Motion selectivity in macaque visual cortex. II. Spatiotemporal range of directional interactions in MT and V1. J. Neurophysiol., 55, 1328-1339.

Muller, R., Gopfert, E., Leineweber, M. \& Greenlee, M.W. (2004) Effect of adaptation direction on the motion VEP and perceived speed of drifting gratings. Vis. Res., 44, 2381-2392.
Nelissen, K., Vanduffel, W. \& Orban, G.A. (2006) Charting the lower superior temporal region, a new motion-sensitive region in monkey superior temporal sulcus. J. Neurosci., 26, 5929-5947.

Niedeggen, M. \& Wist, E.R. (1998) Motion evoked brain potentials parallel the consistency of coherent motion perception in humans. Neurosci. Lett., 246, 61-64.

Niedeggen, M. \& Wist, E.R. (1999) Characteristics of visual evoked potentials generated by motion coherence onset. Cogn. Brain Res., 8, 95-105.

Probst, T., Plendl, H., Paulus, W., Wist, E.R. \& Scherg, M. (1993) Identification of the visual motion area (area V5) in the human brain by dipole source analysis. Exp. Brain Res., 93, 345-351.

Scherg, M. \& Picton, T.W. (1991) Separation and identification of event-related potential components by brain electric source analysis. Electroencephalogr. Clin. Neurophysiol. Suppl., 42, 24-37.

van der Smagt, M.J., Verstraten, F.A. \& van de Grind, W.A. (1999) A new transparent motion aftereffect. Nat. Neurosci., 2, 595-596.

Smith, A.T. \& Edgar, G.K. (1994) Antagonistic comparison of temporal frequency filter outputs as a basis for speed perception. Vis. Res., 34, 253-265.

Thompson, P. (1984) The coding of velocity of movement in the human visual system. Vis. Res., 24, 41-45.

Ungerleider, L.G. \& Mishkin, M. (1982) Two cortical visual systems. In Ingle, D.J., Goodale, M.A. \& Mansfield, R.J.W. (Eds), Analysis of Visual Behavior. MIT Press, Cambridge, MA, pp. 549-586.

Verstraten, F.A.J., van der Smagt, M.J. \& van de Grind, W.A. (1998) Aftereffect of high-speed motion. Perception, 27, 1055-1066.

Verstraten, F.A.J., van der Smagt, M.J., Fredericksen, R.E. \& van de Grind, W.A. (1999) Integration after adaptation to transparent motion: static and dynamic test patterns result in different aftereffect directions. Vis. Res., 39, 803-810.

Wang, J., Zhou, T., Qiu, M., Du, A., Cai, K., Wang, Z., Zhou, C., Meng, M., Zhuo, Y., Fan, S. \& Chen, L. (1999) Relationship between ventral stream for object vision and dorsal stream for spatial vision: an fMRI + ERP study. Hum. Brain Mapp., 8, 170-181.

Zeki, S. (1993) A Vision of the Brain. Blackwell Scientific Publications, Oxford. 